This item was submitted to Loughborough's Research Repository by the author.

Items in Figshare are protected by copyright, with all rights reserved, unless otherwise indicated.

\title{
Cellular senescence: immunosurveillance and future immunotherapy
}

PLEASE CITE THE PUBLISHED VERSION

https://doi.org/10.1016/j.arr.2018.02.001

PUBLISHER

(c) Elsevier

VERSION

AM (Accepted Manuscript)

PUBLISHER STATEMENT

This work is made available according to the conditions of the Creative Commons Attribution-NonCommercialNoDerivatives 4.0 International (CC BY-NC-ND 4.0) licence. Full details of this licence are available at: https://creativecommons.org/licenses/by-nc-nd/4.0/

\section{LICENCE}

CC BY-NC-ND 4.0

\section{REPOSITORY RECORD}

Burton, Dominick, and Alexandra Stolzing. 2019. "Cellular Senescence: Immunosurveillance and Future Immunotherapy". figshare. https://hdl.handle.net/2134/31884. 


\title{
Cellular senescence: immunosurveillance and future immunotherapy
}

Dominick G A Burton* and Alexandra Stolzing

\begin{abstract}
In response to persistent DNA damage, induction into cell senescence promotes an immunogenic program which facilitates immune clearance of these damaged cells. Under physiological conditions, senescent cells can activate both innate and adaptive immune responses, functioning to maintain tissue homeostasis. However, likely owing to ageing of the immune system, a failure to eliminate senescent cells is thought to contribute to their accumulation in tissues with age. As a consequence, senescent cells contribute towards ageing and age-related diseases. Furthermore, induction of cell senescence in immune cells has also been reported, a process which can be both beneficial and detrimental, depending on the biological setting. In addition to activating an immune response, senescent cells have also been reported to promote immunosuppression in pathological contexts. As such, mechanisms associated with senescent cell-immune cell interactions appear complex. In this review, we provide an overview of the research focused on senescence immunosurviellance, including a discussion on the mechanisms by which macrophages may recognise senescent cells. Senescence immunotherapy strategies as an alternative to senolytics for the removal of senescent cells will also be discussed.
\end{abstract}

Key Words: senescence, macrophages, immunosurviellance, ageing, immunotherapy

\author{
*Corresponding author \\ Dr. Dominick Burton \\ Loughborough University, Wolfson School, Epinal Way \\ LE11 3TU, Loughborough, UK \\ d.burton@lboro.ac.uk
}




\section{Introduction}

Clear definitions concerning the biological differences between "cell ageing" and "cell senescence" are often lacking and not well defined. To those unfamiliar with the subject of cellular senescence, such terminology can lead to confusion. We have attempted to briefly clarify such differences. Cellular ageing results from stochastic processes as a result of gradual accumulating damage over time, whereas cellular senescence is a programmed change in cell state associated with permanent growth inhibition. Aged cells maintain their normal function, albeit at a decreased capacity, whereas senescent cells acquire new functions (Figure 1).

In cultures of actively dividing cells, senescence can be abruptly induced in a matter of days. However, in many tissues and organs which consist primarily of quiescent cells (reversible growth arrest) senescence induction can only occur once cells re-enter the cell cycle. The senescent state is often mediated by a persistent DNA damage response (DDR) (Burton and Faragher, 2015; d'Adda di Fagagna, 2008), induced by stress induced stimuli such as telomere dysfunction, oncogene activation, oxidative stress, cell-cell fusion and chemotherapeutic drugs (Chuprin et al., 2013; Di Micco et al., 2006; Ewald et al., 2010; Toussaint et al., 2002; Victorelli and Passos, 2017). "Induction" of senescence therefor refers to the programmed responses which occur following a DDR triggered by a stress induced stimuli. In addition to permanent growth arrest, the senescent state is accompanied by several additional phenotypic changes which make them distinct from their non-senescent counterparts (Table 1). Although a senescence-like response has been reported in post-mitotic cells such as neurons (Jurk et al., 2012), this review will focus on cell senescence in growth-competent cells.

Probably the most widely researched aspect of the senescent phenotype to date is the altered secretory response, referred to as the senescence-messaging secretome, senescence-associated secretory phenotype or the senescence-associated secretome (Campisi and d'Adda di Fagagna, 2007; Kuilman and Peeper, 2009; Malaquin et al., 2016). Here we refer to this response simply as the "senescent secretome". The senescent program is often accompanied with transcriptional changes linked to an altered secretome, consisting of pro-inflammatory cytokines, growth factors and proteases which appear to mimic inflammatory wound repair processes (Coppé et al., 2008; Shelton et al., 1999). In fact, senescent cells have been shown to be important in wound healing, tissue plasticity and tissue regeneration (Demaria et al., 2014; Mosteiro et al., 2016; Ritschka et al., 2017), suggesting that such a secretome is likely physiologically important for such processes. However, to date, the senescent secretome has more often been considered from a pathological perspective, with emphasis being placed on the damaging effects of chronic inflammation in age-related diseases and 
cancer. In recent years there has been a surge of review articles focused on the role of cell senescence in ageing and disease, a subject beyond the scope of this review (Burton and Krizhanovsky, 2014; Childs et al., 2015; Muñoz-Espín and Serrano, 2014; van Deursen, 2014).

With the understanding that senescent cells can be beneficial in the short term, a process that appears to be regulated by immune processes, the concept of the senescent secretome as pathological can be reframed to instead reflect its immunogenic properties, in other words, a secretome that modulates an immune response. Whether a senescent cell is beneficial or detrimental likely depends on how long senescent cells persist within tissues.

Whilst senescent cells secrete factors known to function in the activation, migration, adhesion and differentiation of immune cells in other systems, much more research is needed to investigate the impact of the senescent secretome on specific immune cell functions. Some studies have reported a correlation between the presence of secretory factors associated with cell senescence and immune cell infiltration/localisation within tissues (Kang et al., 2011; Krizhanovsky et al., 2008a; Xue et al., 2007). An overview of factors associated with an immunogenic senescent secretome and their response within different immune cell types has been reviewed previously (Sagiv and Krizhanovsky, 2013).

\section{The senescent program: mechanisms initiating an immune response}

In the majority of instances, induction of the senescence program is initiated by activation of a DDR (d'Adda di Fagagna, 2008). Such a DDR is known to function in the activation of innate immunity and in the development and function of adaptive immunity (Nakad and Schumacher, 2016; Xu, 2006). A DDR during induction of cell senescence appears to be important for inducing an altered secretome capable of attracting and activating immune cells. As later discussed, a DDR has also been shown to be important for upregulating the expression of NKG2D ligands found on senescent cells, enabling their recognition by immune cells (Sagiv et al., 2016).

DNA damage can occur within chromatin packed nuclear DNA leading to activation of transcription factors and other regulators of cell secretion. These include, but are not limited to p38MAPK, NFkB, HMGB1, BRD4, mTOR and GATA4 (Chien et al., 2011; Davalos et al., 2013; Freund et al., 2011; Herranz et al., 2015; Kang et al., 2015; Tasdemir et al., 2016). In addition to nuclear DNA, increased damage to the nucleotide pool has also been shown to induce cell senescence (Rai et al., 2009) and may also involve activation of an immune response. Interestingly, it also appears that DNA damage during induction of cell senescence leads to accumulation of damaged DNA in cytoplasmic foci which 
promotes a senescent secretome. Studies have shown that activation of cyclic GMP-AMP synthase (cGAS), a cytosolic DNA sensor, in senescent cells triggers stimulator of interferon genes (STING) which play an important role in innate immunity (Dou et al., 2017; Glück et al., 2017; Ng et al., 2017; Yang et al., 2017). Mackenzie et al investigated the mechanisms by which cellular DNA gains access to the cytoplasm since it is normally compartmentalised within the nucleus to prevent autoimmunity (Mackenzie et al., 2017). It was reported that cGAS localises to micronuclei arising from genomic instability and that breakdown of the micronuclei envelope exposes self-DNA to the cytosol.

Mechanisms other than a DDR cannot be ruled out. For example, treatment of tumour cells with cyclin-dependent kinase 4 and 6 (CDK4/6) inhibitors were shown to not only induce cell cycle arrest but to also promote anti-tumour immunity (Goel et al., 2017). CDK4/6 inhibition activated the expression of endogenous retroviral elements which stimulated the production of type III interferons leading to enhanced tumour antigen presentation. Rather than activating a DDR, CDK4/6 inhibitors in the context of tumour biology may enhance the susceptibility of such cells to immune checkpoint blockades. However, it should be noted that whilst such cells underwent cell cycle arrest, it does not mean that such cells are senescent and instead may have undergone a state of quiescence (Yoshida and Diehl, 2015).

From a therapeutic perspective, a more in depth understanding of the initial molecular mechanisms involved in activating an immune response is required. Such knowledge may one day be utilised to therapeutically enhance immune clearance of senescent cells as a strategy to alleviate and prevent age-related diseases.

\section{Senescent cells activate an innate immune response}

Scientific research focused on immune surveillance of senescent cells is an emerging field. It has now been approximately ten years since the first evidence for such a response was reported. In that study, Xue et al demonstrated that reactivation of p53 in p53-deficient tumours promoted induction of a cellular senescence program (Xue et al., 2007). This was associated with activation of an innate immune system as evident by increased expression of transcripts specific to natural killer (NK) cells, macrophages and neutrophils. This immune activation consequently resulted in tumour clearance, suggesting that the senescence program functions to prevent cancer development. It also suggested that induction of cell senescence rather than cell death may prove a more effective strategy for targeting cancers. 
The following year, Krizhanovsky et al reported another benefit of immune clearance of senescent cells: the resolution of liver fibrosis following damage (Krizhanovsky et al., 2008b). Immune cells such as NK cells, macrophages and neutrophils were found to be in proximity to senescent cells in fibrotic livers. In addition, senescent activated stellate cells generated during liver damage were shown to be preferentially killed by NK cells both in vitro and in vivo. In doing so, immune clearance of senescent cells prevented excess fibrosis which occurred when cells were unable to enter the senescent state. Other than possibly acting as an anti-cancer mechanism, senescent cells until this point were frequently considered as only detrimental to the tissue in which they reside. However, it is now apparent that senescent cells could also play beneficial roles and that this is likely dependent upon the biological context. Following on from these findings, a role for cell senescence in other fibrotic models were also reported (Fitzner et al., 2012). In addition, physiological functions for senescent cells such as during wound healing, regeneration, embryonic/placental development and skeletal bone regulation during late puberty, slowly began to emerge (Chuprin et al., 2013; Demaria et al., 2014; Li et al., 2017; Muñoz-Espín et al., 2013a; Storer et al., 2013; Yun et al., 2015). However, the possibility that the aforementioned studies are instead reporting non-senescent cells displaying biomarkers in common with senescent cells cannot be ruled out.

Sagiv et al later investigated the mechanisms promoting NK mediated killing of senescent cells. Granule exocytosis, but not death-receptor-mediated apoptosis, was shown to be required for NK cellmediated killing of senescent cells (Sagiv et al., 2013). Mice with defects in granule exocytosis accumulated senescent stellate cells within the liver following damage leading to increased fibrosis. In a follow-up study, Sagiv et al also investigated the mechanisms by which NK cells specifically recognise and target senescent cells. Ligands of an activating Natural Killer (NK) cell receptor (NKG2D), MICA and ULBP2 were found to be consistently upregulated following induction of replicative senescence, oncogene-induced senescence, etoposide-induced senescence and were found to be required for efficient NK-mediated cytotoxicity towards senescent cells (Sagiv et al., 2016). During liver fibrosis, senescent activated stellate cells were found to accumulate in mice lacking the NKG2D receptor. Other studies have reported the upregulation of NKG2D ligands following induction of cell senescence in tumour cells (Antonangeli et al., 2016; lannello et al., 2013).

Whilst mechanistically, NK cells have been the primary focus to date in regard to immune surveillance of senescent cells, additional innate immune cells also appear important. Lujambio et al went on to publish findings focused on macrophage responses towards senescent cells. This study demonstrated that p53-expressing senescent stellate cells release factors which promote macrophage polarisation towards a tumour-inhibiting M1 state capable of targeting senescent cells in cultures (Lujambio et al., 2013). Findings by Hall et al suggest that alginate-encapsulated senescent cells may induce a M2-like 
phenotype in peritoneal macrophages of mice as evident by elevated expression of arginase 1 (Arg1) (Hall et al., 2016). M2 macrophages are also capable of phagocytosis and so have the potential to also induce cell death in senescent cells. In addition, the induction of macrophages into $M 2 a, M 2 b$, and $\mathrm{M} 2 \mathrm{c}$ subtypes by senescent cells may also occur and so further investigations are required. Other studies have also suggested that macrophages play a role in immune surveillance of senescent cells. An earlier study by Hall et al showed that alginate beads containing senescent cells promote macrophage recruitment when transplanted into mice (Hall et al., 2016). Findings from Yun et al, who has pioneered cell senescence research within the salamander model, suggests that macrophages are important for efficient clearance of senescent cells during salamander limb regeneration (Yun et al., 2015). Interestingly, macrophages may also promote induction of cell senescence in tumour cells as a possible anti-cancer mechanism (Reimann et al., 2010).

\section{Senescent cells induce an adaptive immune response}

In 2011, Kang et al demonstrated that pre-malignant senescent hepatocytes undergo immunemediated clearance by CD4+ T cells (Kang et al., 2011). The presence of major histocompatibility complex class II (MHCII) molecules on senescent hepatocytes appeared to be important for direct killing of pre-malignant senescent hepatocytes. However, $\mathrm{MHCll}$ expression was not sufficient for inducing proliferation of naïve $\mathrm{CD} 4^{+} \mathrm{T}$ cells. In addition, killing of pre-malignant senescent hepatocytes by $\mathrm{CD} 4^{+} \mathrm{T}$ cells required the presence of monocytes/macrophages. Macrophage depletion in mice via gadolinium treatment greatly reduced immune surveillance of senescent cells. An impairment in immune clearance of pre-malignant senescent hepatocytes resulted in the development of hepatocellular carcinomas. This study again demonstrated the importance of immune surveillance of senescent cells in tumour suppression. Whilst senescent cells may promote T cell recruitment for their clearance, $T$ cells may also induce cell senescence in cancer cells as a mechanism to limit cancer progression (Braumüller et al., 2013; Rakhra et al., 2010).

Another study also suggests that oncogene-induced senescence (OIS) in primary human melanocytes can activate aspects of the adaptive immune response. van Tuyn et al have shown that during OIS, melanocytes express MHC class II antigen presentation molecules which can activate T cell proliferation in vitro (van Tuyn et al., 2017). In vivo, non-proliferating, oncogene expressing melanocytes localize to skin-draining lymph nodes where they induce $T$ cell proliferation and an antigen presentation gene expression signature. Senescent melanocytes are thought to form within 
benign melanocytic nevi which are known to persist for decades (Joselow et al., 2017), suggesting that immune surveillance mechanisms are not active. The authors of this study suggest that benign human nevi represent a subset of OIS melanocytes that have been selected for downregulation of MHC II via evasion of MHC II-mediated immune-editing, providing a possible explanation for their persistence.

Immune surveillance of senescent cells during reproductive processes

In recent years there has been emerging interest in the role of cell senescence during processes associated with pregnancy. In 2013, three papers were published demonstrating a potential role for senescent cells in embryonic and placental development (Chuprin et al., 2013; Muñoz-Espín et al., 2013b; Storer et al., 2013). Two back-to-back papers published in Cell suggested that senescent-like cells function to promote tissue remodelling during embryonic growth and patterning in mice, a process which appears to be associated with macrophage clearance (Muñoz-Espín et al., 2013a; Storer et al., 2013).

In a study focused on placental development, fusion-induced cell senescence was reported to function in the formation of the syncytiotrophoblast, which serves as the maternal/foetal interface at the placenta (Chuprin et al., 2013). Fusion-induced senescence promoted the upregulation of secretory components associated with immune surveillance. It was suggested that cytokines secreted from these senescent cells may function in placental growth during pregnancy, protecting the foetus from pathological organisms and facilitating immune cell interaction (Burton and Krizhanovsky, 2014).

More recently, Egashira et al reported that macrophages contribute towards the clearance of senescent cells in the mouse postpartum uterus (Egashira et al., 2017). Depletion of macrophages in the postpartum mice increased the number of senescent cells in the uterus. In p53-deleted mice, uterine senescent cells increased with the authors suggesting that uterine p53 deficiency may affect the distribution of a macrophage subpopulation, leading to impaired clearance of senescent cells. However, p53-deleted mouse models are often used in studies to prevent cell senescence, so an increase in this instance is perplexing and requires further study. Excessive uterine senescent cells were associated with a decrease in second pregnancy success rate in a preterm birth model. In another study, findings by Brighton et al suggest that acute decidual cell senescence governs endometrial rejuvenation and remodelling at embryo transplantation (Brighton et al., 2017). During this process, uterine natural killer cells were shown to selectively target and clear senescent decidual cells through granule exocytosis. 
Although the senescent programme may play important physiological functions during pregnancy, under other contexts it may contribute to pathological processes during pregnancy (reviewed in Velarde \& Menon 2016; Cox \& Redman 2017).

\section{Cellular senescence: immunosuppressive responses}

Whilst a number of studies have demonstrated a positive physiological impact by which senescent cells promote immune clearance, other studies have suggested that a senescence-mediated immune response can also be immunosuppressive and tumour promoting. In 2014, a study conducted by Di Mitri et al demonstrated that senescence induction in PTEN null prostate tumours in mice promoted an infiltration of Gr-1+ myeloid cells which protected a fraction of proliferating tumour cells from cell senescence, thereby sustaining tumour growth (Di Mitri et al., 2014). During the same year the another study was published reporting that Pten-loss induced cell senescence in tumours promoted an immunosuppressive secretome that contributed to tumour growth and chemoresistance (Toso et al., 2014).

Ruhland et al also went on to provide evidence suggesting that senescent cells can establish an immunosuppressive microenvironment that drives tumorigenesis (Ruhland et al., 2016). In this study, senescent stromal cells were shown to drive localised increases in suppressive myeloid cells that contributed to tumour promotion. In particular, interleukin-6, a common component of the senescent secretome, was responsible for inducing the increase in suppressive myeloid cells and their ability to inhibit anti-tumour T cell responses.

In the same year, Eggert et al reported that senescence-associated immune responses can both promote tumour suppression and tumour progression depending on the context (Eggert et al., 2016). The CCL2-CCR2 axis was shown to mediate myeloid cell accumulation in mice livers following induction of senescent cells, which acted as tumour suppressive in the early stages of liver tumorigenesis. However, when senescent cells are present in later phases of tumour development, the combination of senescent cells and tumour cells instead promotes tumour progression. The senescent secretome in this instance promoted the growth of liver cancer by inhibiting NK cell function through the accumulation of immunosuppressive immature myeloid cells.

Whilst much more research is required to determine the mechanisms promoting an immunosuppressive phenotype in senescent cells, it is likely dependent upon the genetic background of the cells, the mode of senescence induction and whether senescent cells have persisted and 
undergone changes that may be attributed to a chronic response rather than a beneficial acute response. Experimentally underexplored is the concept of acute and chronic cell senescence. Acute senescence, referring to the early activation of the senescence program for a limited time-frame is likely beneficial, functioning in physiological processes. However, if senescent cells are not removed, they may develop a chronic phenotype associated with persistent inflammation which promotes agerelated inflammatory diseases. In the cancer microenvironment, chronic inflammation can promote an immunosuppressive environment (Wang and DuBois, 2015), a process that likely extends to a senescence microenvironment.

\section{Cell senescence of immune cells}

Cell senescence of immune cells should not be confused with immunosenescence, the ageing of the immune system, which although may include induction of cell senescence, also includes an array of age-related changes unrelated to cell senescence. It is also important to note that the term "senescent" is often used interchangeable with "aged" by immunologists discussing immune cell ageing (see figure 1 for our definition of the terminology). The absence of separation regarding genuine mechanisms of immune cell ageing with those of the cell senescent program can lead to confusion. An awareness of immune cell senescence is important because such changes likely impact their response towards senescent cells of non-immune origin.

T cells: The majority of research to date focused on immune cell senescence has been undertaken on T cells. Like cell senescence in non-immune cells, T cell senescence is associated with irreversible growth arrest and a pro-inflammatory secretome that can contribute towards ageing and age-related diseases (Chou and Effros, 2013). However, the possibility that the senescence program may also be utilised by $T$ cells during physiological immune responses cannot be ruled out.

Regulatory T cells (Tregs) play a role in regulating or suppressing other cells of the immune system. Ye et al has demonstrated that one way in which Tregs appear to promote immune suppression is through the induction of cell senescence in responder naïve and effector T cells (Ye et al., 2012). In addition, Montes et al reported that soluble factors from tumours can induce human T cells to develop a senescent-like phenotype that is also associated with the suppression of responder $\mathrm{T}$ cell proliferation (Montes et al., 2008). Such an immunosuppressive response likely functions in both physiological and pathological processes depending on the biological context. For example, Tregs have been shown to beneficial through facilitating cutaneous wound healing (Nosbaum et al., 2016), 
but can also be detrimental by promoting tumour development (Beyer and Schultze, 2006). Cancer can be considered as a persistent wound healing response (Schäfer and Werner, 2008), which may explain the role of immune cells in both these contexts. Ramello et al went on to demonstrate that senescent $T$ cells also promote the secretion of pro-inflammatory cytokines and angiogenic factors by human monocytes/macrophages (Ramello et al., 2014).

Natural Killer cells: In addition to T cells, benefits of NK cell senescence have been reported. Rajagopalan and Long have provided evidence to suggest that induction of NK cell senescence by CD158d functions to reprogram NK cells in order to promote vascular remodelling during pregnancy (Rajagopalan and Long, 2012). CD158d, a receptor for soluble HLA-G (produced by foetal trophoblast cells during early pregnancy) was shown to trigger a DDR in NK cells leading to the appearance of biomarkers associated with cell senescence, including p21 expression and a senescent secretome. This senescent secretome promoted vascular remodelling and angiogenesis, indicating that NK cell senescence in this instance is beneficial for favouring reproduction. However, tissue remodelling and angiogenesis mediated by a senescent secretome in other contexts can be detrimental (Oubaha et al., 2016).

Astrocytes: Biomarkers of cell senescence have also been observed in response to hydrogen peroxide treatment in human and rodent astrocytes (Bitto et al., 2010). Stress-induced astrocytes in culture underwent growth arrest, morphological alterations, expressed SA-beta-Gal activity and increased p21, p53 and p16 expression. A similar finding was observed for astrocytes following extended cell culture. In a follow-up study, Crowe et al monitored the transcriptional changes associated with stress-induced senescence in human astrocytes, reporting that brain-expressed genes, including those involved in neuronal development and differentiation, were down-regulated in senescent astrocytes (Crowe et al., 2016). Astrocyte senescence also appears to occur in vivo. Bhat et al assessed biomarkers of cell senescence in astrocytes of brain tissue from aged individuals and patients with Alzheimer's disease (AD) (Bhat et al., 2012). A significant increase in p16 ${ }^{\text {INK4A }}$-positive astrocytes was observed in subjects aged 35-50 years and 78-90 years when compared with foetal tissue samples. In culture, it was also shown that beta-amyloid could trigger cell senescence, suggesting that senescent astrocytes may increase the risk of sporadic AD. In another study related to HIV therapy, it was reported that highly active antiretroviral therapy (HAART) increases biomarkers associated with cell senescence (cell cycle arrest, SA-Beta-Gal activity, p21 expression) in primary cultures of human astrocytes (Cohen et al., 2017). It was suggested that HAART-induced senescent astrocytes may play a role in neurocognitive impairment observed in HIV-infected patients. 
Macrophages: Evidence for cell senescence in macrophages to date is less convincing. In response to hyperoxia $\left(95 \% \mathrm{O}_{2}\right)$, RAW264.7 macrophages have been shown to undergo cell cycle arrest associated with increases in $\mathrm{p} 21^{\mathrm{CIP} 1}$ and $\mathrm{p} 53$ accumulation, characteristic features of cell senescence (Nyunoya et al., 2003). In another study, acrylamide was shown to induce a senescence-like response in RAW264.7 and mouse peritoneal macrophages, including cell cycle arrest, altered morphology and SA-Beta-Gal activity (Kim et al., 2015). Progressive telomere shortening and induction of cell cycle arrest has also been observed in rat microglia cells, a type of macrophage found in the brain (Flanary and Streit, 2004). However, whilst it can be assumed that cell cycle arrest in this instance is related to cell senescence, further characterisation is required. Caldeira et al have shown that after a very short period (16 Days) in culture, mouse microglia cells displayed altered morphology and increased SAbeta-Gal activity typical of senescent cells (Caldeira et al., 2014). Interestingly, NFkB activity which is normally elevated in non-immune senescent cells was decreased in culturally aged microglia cells in this instance. However, analysis of cell cycle does not appear to have been undertaken and since SAbeta-Gal activity has been observed in culturally aged but growth competent cells (Walters et al., 2016), it cannot be assumed that such cells are senescent. Finally, SA-beta-Gal activity and p16 INKAA $^{\text {I }}$ expression in macrophages has also been reported to be elevated in ageing mice, with the authors suggesting that such cells are a subclass of macrophages rather than being senescent (Hall et al., 2016).

\section{Recognition of senescent cells by macrophages: possible mechanisms}

Whilst there is currently little understanding concerning the mechanisms governing macrophage mediated recognition of senescent cells, the processes are probably not specific to senescent cells. Rather, the more characterised molecular mechanisms associated with macrophage recognition in other cell models such as during cancer immunosurveillance and apoptotic cell clearance may also be pertinent for senescence surveillance.

Apoptotic cells have been shown to preferentially express specific cell surface antigens which can be recognised by naturally occurring antibodies (IgMs) that enable phagocytosis by macrophages. As such, it can be speculated that senescent cells may also express specific cell surface antigens which would not only provide insights into the mechanisms mediating immune clearance, but would also provide a means to specifically identify senescent cells in tissues. In a study focused on identifying antibodies which specifically target senescent cells, researchers identified the IgM clone 9H4 which recognises an oxidised form of membrane-bound vimentin (malondialdehyde (MDA) -modified 
vimentin) expressed on the surface of senescent human fibroblasts (Frescas et al., 2017). Whilst not the focus of the study, the authors speculate that MDA-modified vimentin may serve as an "eat me" signal leading to phagocytosis by macrophages (Figure 2).

Senescent cells commonly display higher levels of ROS, likely owing to changes in cell metabolism and/or the presence of dysfunctional mitochondria, which can lead to the oxidation of phospholipids (Ademowo et al., 2017). During apoptosis, oxidatively modified lipids on the surface of cell membranes can function as pattern recognition ligands promoting macrophage recognition and phagocytosis via the scavenger receptor CD36 (Hazen, 2008), RAGE (Friggeri et al., 2011) and other class I and class II scavenger receptors (Canton et al., 2013). Macrophage CD36 binds to and internalises oxidised phospholipids and lipoproteins (Silverstein and Febbraio, 2009). Whether CD36 plays a role in the senescence surveillance by macrophages has yet to be determined (Figure 2). The naturally occurring antibody EO6 is also known to specifically recognise oxidised phospholipids, functioning as an "eat me" signal for macrophages. Whilst this occurs during apoptosis, it raises the possibility that it may also function as a mechanism promoting senescence immunosurveillance by macrophages. However, Frescas et al (2017) claim that E06 failed to recognise senescent cells, but this may depend on how long cells have been senescent and so further research is required.

Surface expression of CD47 acts as a "don't eat me" signal, sending inhibitory signals through SIRPa, a receptor expressed on the surface of macrophage, ensuring that healthy cells are not inappropriately phagocytosed. Therefore, the downregulation of CD47 would be required for macrophages to target damaged "self" cells (Liu et al., 2017). One study has demonstrated that induction of tumour cell senescence via c-Myc inactivation leads to the downregulation of CD47 which consequently promoted tumour regression (Casey et al., 2016). Whether CD47 downregulation in this instance is a specific response to c-Myc inactivation or activation of the senescence program is unclear. However, it would make biological sense to downregulate CD47 during cell senescence to enable removal of damaged "self" cells, but further research is required (Figure 2). In addition to CD47 in this study, the downregulation of programmed death-ligand 1 (PD-L1), a protein thought to play a role in immune suppression (Kleinovink et al, 2017), was also observed upon c-Myc inactivation-induced senescence.

Modified cell surface glycan's are also potential senescence antigens that can be recognised by the Ctype lectin receptor (CLR) family (Figure 2). One of the CLRs exclusively expressed by macrophages is the macrophage galactose-type lectin (MGL) (van Kooyk et al., 2015). N- and O-glycan surface protein modifications have been associated with senescent fibroblasts with an observed senescenceassociated decrease in sialylation and increase in galactose exposure (Itakura et al., 2016). The 
expression of the $\alpha-2,6$-sialyltransferase I (ST6Gal I), which transfers sialic acid to galactose residues of $\mathrm{N}$-glycans has also been shown to decrease during in vitro cell ageing (Tadokoro et al., 2006).

\section{Beyond Senolytics: immunotherapy targeting senescent cells}

Immunotherapeutic strategies already in development for combating cancer may one day be repurposed for targeting senescent cells for the alleviation of age-related diseases (see Figure 3). Discussed in detail elsewhere (Burton and Krizhanovsky, 2014; Childs et al., 2015; Muñoz-Espín and Serrano, 2014; van Deursen, 2014), such age-related diseases include diabetes, Alzheimer's, pulmonary fibrosis, cardiovascular disease and osteoporosis. In addition, identifying further molecular changes associated with senescent cells, especially cell-type specific alterations, would be advantageous for developing therapeutic approaches for targeting senescent cells. However, since senescent cells can also be beneficial, the elimination of acute senescent cells could be problematic. Therefore, the identification of therapeutic targets specific to chronic senescent which are absent in acute senescent cells would be highly desirable.

A recent study identified the expression of CD26/dipeptidyl peptidase 4 (DPP4) on the membrane surface of senescent fibroblasts which have undergone replicative senescence (Kim et al, 2017). This study assessed the potential use of DPP4 as a membrane target for promoting antibody-dependent cell-mediated cytotoxicity (ADCC) against senescent cells. ADCC is a natural mechanism of cellmediated immune defence involving the activation NK cells by antibodies which has been adapted to promote NK cell-mediated ADCC in cancer immunotherapy (Wang et al., 2015). Kim et al demonstrated that targeting DPP4 via an ADCC assay in vitro preferentially sensitised senescent, but not proliferating cells to cytotoxicity by NK cells. These finding highlight a potential immunotherapeutic strategy for targeting senescent cells.

As stated previously, one of the mechanisms by which NK cells specifically recognise and kill senescent cells is via the surface expression of NKG2D ligands (Sagiv et al., 2016). Since many tumour cells also express NKG2D ligands, such ligands have been suggested to be a useful target for immunotherapeutic approaches in cancer (Spear et al., 2013), and so could be adapted for senescent cell clearance. For example, the use of engineered immune cells such as chimeric antigen receptor (CAR) T cells to target specific molecules on cancer cells has great potential as an anti-cancer therapy (Yu et al., 2017). As such, it may be possible to target senescent cells by engineering T cells to express a NKG2D CAR which recognise NKG2D ligands on the surface of senescent cells. 
An adaption of cancer vaccines could also be considered for boosting immune clearance of senescent cells. Although a universal biomarker of cell senescence has not been identified, the exposure of senescent cell membranes to immune cells may evoke an immune response to antigens not yet identified. In one approach, senescence vaccines would involve the isolation of senescence specific antigens (SSAs) which are then exposed to dendritic cells, professional antigen presenting cells. In response to SSA uptake, dendritic cells process and express these antigens on their cell surface which can then be recognised by $T$ cells. T cell interaction with these antigens promotes $T$ cell activation, differentiation and ultimately killing of target cells.

As discussed previously, the senescence program can also be activated in immune cells, which likely contributes towards impaired immune function, contributing to senescent cell accumulation. In addition to impaired immune cell function, the persistence of senescent immune cells would likely take up valuable immunological space required for expansion of functionally competent immune cells. T cells for example undergo rapid expansion in response to antigenic stimuli followed by cell-mediated apoptosis (Chou and Effros, 2013), a process that may be prevented if senescent T cells are present. As such, another strategy for promoting the elimination of non-immune senescent cells may be the removal of senescent immune cells with the anticipation that such an approach would enhance immune function.

One possibility could be to filter the blood of the unwanted senescent immune cells, maybe through the use of antibodies linked to magnetic nanoparticles/beads (Chen et al., 2017; Rebo et al., 2010). Another strategy may be the use of senolytic drugs to specifically kill senescent immune cells or senescent hematopoietic stem cells (HSCs) which give rise to immune cells. Senolytic targeting of senescent HSCs in mice leading to rejuvenation of aged tissue stem cells has already provided promising results (Chang et al., 2015). Rather than eliminating senescent immune cells, it may one day be possible to rejuvenate senescent immune cells, reversing their detrimental phenotype to improve immune function. For example, inhibition of p38 signalling in CD8+ T cells which exhibit features of cell senescence, increased proliferation, telomerase activity, mitochondria biogenesis and fitness (Henson et al., 2014). Small molecules based on resveratrol have also been reported to rescue cells from cell senescence (Latorre et al., 2017).

The ability of immune cells to migrate and kill target cells can become compromised with age, suggesting that therapeutically boosting these responses may be beneficial for promoting senescent cell clearance. One approach that can be adapted within immune cells is the engineering of cells that recognise specific components of the senescent secretome. As proof of principle, Qudrat et al generated cells with a chimeric IL-6 receptor (IL6Rchi) that generates a $\mathrm{Ca}^{2+}$ signal in response to IL-6, 
a component of the senescent secretome (Qudrat et al., 2017). When IL6Rchi was expressed with an engineered $\mathrm{Ca}^{2+}$-activated RhoA (CaRQ), it enabled directed migration to IL-6 in cells which normally have no such function. Furthermore, the expression of vesicular stomatitis virus glycoprotein $G$ (VSVG) and herpes simplex virus type 1 thymidine kinase (TK) in these IL-6 seeking cells allowed cellcell fusion to occur with target cells and consequently cell death upon administration of ganciclovir, an anti-viral drug.

\section{Concluding remarks}

Senescent cells can be both beneficial and detrimental. Therefore, any therapeutic targeting of senescent cells needs to take into consideration that such approaches may also be harmful. This may include senescent cell removal during pregnancy or injury. However, some findings suggest that acute senescence mechanisms may be preserved during lifelong removal of senescent cells in naturally aged mice, but further research is required (Baker et al., 2016). Another aspect to consider is the biological consequences to mass removal of senescent cells. Such mass removal may disrupt tissue integrity and/or activate a strong pro-inflammatory response depending on the mode of cell death. Other therapeutic strategies in conjunction with senescent cell removal such as implantation of stem cells to aid repair may have to be considered (Burton, 2009). Whilst, there is currently more concern with therapeutically eliminating senescent cells once they have appeared, future strategies focused on preventing their long-term persistence would be greatly desirable.

\section{References}


Ademowo, O.S., Dias, H.K.I., Burton, D.G.A., Griffiths, H.R., 2017. Lipid (per) oxidation in mitochondria: an emerging target in the ageing process? Biogerontology. https://doi.org/10.1007/s10522-017-9710-z

Antonangeli, F., Soriani, A., Ricci, B., Ponzetta, A., Benigni, G., Morrone, S., Bernardini, G., Santoni, A., 2016. Natural killer cell recognition of in vivo drug-induced senescent multiple myeloma cells. Oncoimmunology 5, e1218105. https://doi.org/10.1080/2162402X.2016.1218105

Baker, D.J., Childs, B.G., Durik, M., Wijers, M.E., Sieben, C.J., Zhong, J., A. Saltness, R., Jeganathan, K.B., Verzosa, G.C., Pezeshki, A., Khazaie, K., Miller, J.D., van Deursen, J.M., 2016. Naturally occurring p16Ink4a-positive cells shorten healthy lifespan. Nature 530, 184-189. https://doi.org/10.1038/nature16932

Beyer, M., Schultze, J.L., 2006. Regulatory T cells in cancer. Blood 108, 804-811. https://doi.org/10.1182/blood-2006-02-002774

Bhat, R., Crowe, E.P., Bitto, A., Moh, M., Katsetos, C.D., Garcia, F.U., Johnson, F.B., Trojanowski, J.Q., Sell, C., Torres, C., 2012. Astrocyte Senescence as a Component of Alzheimer's Disease. doi.org 7, e45069. https://doi.org/10.1371/journal.pone.0045069

Bitto, A., Sell, C., Crowe, E., Lorenzini, A., Malaguti, M., Hrelia, S., Torres, C., 2010. Stress-induced senescence in human and rodent astrocytes. Exp. Cell Res. 316, 2961-8. https://doi.org/10.1016/j.yexcr.2010.06.021

Braumüller, H., Wieder, T., Brenner, E., Aßmann, S., Hahn, M., Alkhaled, M., Schilbach, K., Essmann, F., Kneilling, M., Griessinger, C., Ranta, F., Ullrich, S., Mocikat, R., Braungart, K., Mehra, T., Fehrenbacher, B., Berdel, J., Niessner, H., Meier, F., van den Broek, M., Häring, H.-U., Handgretinger, R., Quintanilla-Martinez, L., Fend, F., Pesic, M., Bauer, J., Zender, L., Schaller, M., Schulze-Osthoff, K., Röcken, M., 2013. T-helper-1-cell cytokines drive cancer into senescence. Nature 494, 361-365. https://doi.org/10.1038/nature11824

Brighton, P.J., Maruyama, Y., Fishwick, K., Vrljicak, P., Tewary, S., Fujihara, R., Muter, J., Lucas, E.S., Yamada, T., Woods, L., Lucciola, R., Lee, Y.H., Takeda, S., Ott, S., Hemberger, M., Quenby, S., Brosens, J.J., 2017. Clearance of senescent decidual cells by uterine natural killer cells drives endometrial remodeling during the window of implantation. doi.org 172593. https://doi.org/10.1101/172593

Burton, D.G.A., 2009. Cellular senescence, ageing and disease. Age (Omaha). 31, 1-9. https://doi.org/10.1007/s11357-008-9075-y 
Burton, D.G.A., Faragher, R.G.A., 2015. Cellular senescence: from growth arrest to immunogenic conversion. Age (Omaha). 37, 9764. https://doi.org/10.1007/s11357-015-9764-2

Burton, D.G., Krizhanovsky, V., 2014. Physiological and pathological consequences of cellular senescence. https://doi.org/10.1007/s00018-014-1691-3

Caldeira, C., Oliveira, A.F., Cunha, C., Vaz, A.R., FalcÃfo, A.S., Fernandes, A., Brites, D., 2014. Microglia change from a reactive to an age-like phenotype with the time in culture. Front. Cell. Neurosci. 8. https://doi.org/10.3389/fncel.2014.00152

Campisi, J., d'Adda di Fagagna, F., 2007. Cellular senescence: when bad things happen to good cells. Nat. Rev. Mol. Cell Biol. 8, 729-740. https://doi.org/10.1038/nrm2233

Canton, J., Neculai, D., Grinstein, S., 2013. Scavenger receptors in homeostasis and immunity. Nat. Rev. Immunol. 13, 621-634. https://doi.org/10.1038/nri3515

Casey, S.C., Tong, L., Li, Y., Do, R., Walz, S., Fitzgerald, K.N., Gouw, A.M., Baylot, V., Gutgemann, I., Eilers, M., Felsher, D.W., 2016. MYC regulates the antitumor immune response through CD47 and PD-L1. Science (80-. ). 352, 227-231. https://doi.org/10.1126/science.aac9935

Chang, J., Wang, Y., Shao, L., Laberge, R.-M., Demaria, M., Campisi, J., Janakiraman, K., Sharpless, N.E., Ding, S., Feng, W., Luo, Y., Wang, X., Aykin-Burns, N., Krager, K., Ponnappan, U., HauerJensen, M., Meng, A., Zhou, D., 2015. Clearance of senescent cells by ABT263 rejuvenates aged hematopoietic stem cells in mice. Nat. Med. 22, 78-83. https://doi.org/10.1038/nm.4010

Chen, J., Chen, K.-H., Fu, B.-Q., Zhang, W., Dai, H., Lin, L.-R., Wang, L.-M., He, Y.-N., 2017. Isolation and identification of senescent renal tubular epithelial cells using immunomagnetic beads based on DcR2. Exp. Gerontol. 95, 116-127. https://doi.org/10.1016/j.exger.2017.04.008

Chien, Y., Scuoppo, C., Wang, X., Fang, X., Balgley, B., Bolden, J.E., Premsrirut, P., Luo, W., Chicas, A., Lee, C.S., Kogan, S.C., Lowe, S.W., 2011. Control of the senescence-associated secretory phenotype by NF-KB promotes senescence and enhances chemosensitivity. Genes Dev. 25, 2125-36. https://doi.org/10.1101/gad.17276711

Childs, B.G., Durik, M., Baker, D.J., van Deursen, J.M., 2015. Cellular senescence in aging and agerelated disease: from mechanisms to therapy. Nat. Med. 21, 1424-1435. https://doi.org/10.1038/nm.4000

Chou, J.P., Effros, R.B., 2013. T cell replicative senescence in human aging. Curr. Pharm. Des. 19, 1680-98. 
Chuprin, A., Gal, H., Biron-Shental, T., Biran, A., Amiel, A., Rozenblatt, S., Krizhanovsky, V., 2013. Cell fusion induced by ERVWE1 or measles virus causes cellular senescence. Genes Dev. 27, 235666. https://doi.org/10.1101/gad.227512.113

Cohen, J., D'Agostino, L., Wilson, J., Tuzer, F., Torres, C., 2017. Astrocyte Senescence and Metabolic Changes in Response to HIV Antiretroviral Therapy Drugs. Front. Aging Neurosci. 9, 281. https://doi.org/10.3389/fnagi.2017.00281

Coppé, J.-P., Patil, C.K., Rodier, F., Sun, Y., Muñoz, D.P., Goldstein, J., Nelson, P.S., Desprez, P.-Y., Campisi, J., 2008. Senescence-associated secretory phenotypes reveal cell-nonautonomous functions of oncogenic RAS and the p53 tumor suppressor. PLoS Biol. 6, 2853-68. https://doi.org/10.1371/journal.pbio.0060301

Cox, L.S., Redman, C., 2017. The role of cellular senescence in ageing of the placenta. Placenta 52, 139-145. https://doi.org/10.1016/j.placenta.2017.01.116

Crowe, E.P., Tuzer, F., Gregory, B.D., Donahue, G., Gosai, S.J., Cohen, J., Leung, Y.Y., Yetkin, E., Nativio, R., Wang, L.-S., Sell, C., Bonini, N.M., Berger, S.L., Johnson, F.B., Torres, C., 2016. Changes in the Transcriptome of Human Astrocytes Accompanying Oxidative Stress-Induced Senescence. Front. Aging Neurosci. 8, 208. https://doi.org/10.3389/fnagi.2016.00208

d'Adda di Fagagna, F., 2008. Living on a break: cellular senescence as a DNA-damage response. Nat. Rev. Cancer 8, 512-522. https://doi.org/10.1038/nrc2440

Davalos, A.R., Kawahara, M., Malhotra, G.K., Schaum, N., Huang, J., Ved, U., Beausejour, C.M., Coppe, J.-P., Rodier, F., Campisi, J., 2013. p53-dependent release of Alarmin HMGB1 is a central mediator of senescent phenotypes. J. Cell Biol. 201, 613-629. https://doi.org/10.1083/jcb.201206006

Demaria, M., Ohtani, N., Youssef, S.A., Rodier, F., Toussaint, W., Mitchell, J.R., Laberge, R.-M., Vijg, J., Van Steeg, H., Dollé, M.E.T., Hoeijmakers, J.H.J., de Bruin, A., Hara, E., Campisi, J., 2014. An Essential Role for Senescent Cells in Optimal Wound Healing through Secretion of PDGF-AA. Dev. Cell 31, 722-733. https://doi.org/10.1016/j.devcel.2014.11.012

Di Micco, R., Fumagalli, M., Cicalese, A., Piccinin, S., Gasparini, P., Luise, C., Schurra, C., Garre', M., Nuciforo, P.G., Bensimon, A., Maestro, R., Pelicci, P.G., d’Adda di Fagagna, F., 2006. Oncogeneinduced senescence is a DNA damage response triggered by DNA hyper-replication. Nature 444, 638-42. https://doi.org/10.1038/nature05327

Di Mitri, D., Toso, A., Chen, J.J., Sarti, M., Pinton, S., Jost, T.R., D’Antuono, R., Montani, E., Garcia- 
Escudero, R., Guccini, I., Da Silva-Alvarez, S., Collado, M., Eisenberger, M., Zhang, Z., Catapano, C., Grassi, F., Alimonti, A., 2014. Tumour-infiltrating Gr-1+ myeloid cells antagonize senescence in cancer. Nature 515, 134-137. https://doi.org/10.1038/nature13638

Dou, Z., Ghosh, K., Vizioli, M.G., Zhu, J., Sen, P., Wangensteen, K.J., Simithy, J., Lan, Y., Lin, Y., Zhou, Z., Capell, B.C., Xu, C., Xu, M., Kieckhaefer, J.E., Jiang, T., Shoshkes-Carmel, M., Tanim, K.M.A. Al, Barber, G.N., Seykora, J.T., Millar, S.E., Kaestner, K.H., Garcia, B.A., Adams, P.D., Berger, S.L., 2017. Cytoplasmic chromatin triggers inflammation in senescence and cancer. Nature 550, 402-406. https://doi.org/10.1038/nature24050

Egashira, M., Hirota, Y., Shimizu-Hirota, R., Saito-Fujita, T., Haraguchi, H., Matsumoto, L., Matsuo, M., Hiraoka, T., Tanaka, T., Akaeda, S., Takehisa, C., Saito-Kanatani, M., Maeda, K.-I., Fujii, T., Osuga, Y., 2017. F4/80+ Macrophages Contribute to Clearance of Senescent Cells in the Mouse Postpartum Uterus. Endocrinology 158, 2344-2353. https://doi.org/10.1210/en.2016-1886

Eggert, T., Wolter, K., Ji, J., Ma, C., Yevsa, T., Klotz, S., Medina-Echeverz, J., Longerich, T., Forgues, M., Reisinger, F., Heikenwalder, M., Wang, X.W., Zender, L., Greten, T.F., 2016. Distinct Functions of Senescence-Associated Immune Responses in Liver Tumor Surveillance and Tumor Progression. Cancer Cell 30, 533-547. https://doi.org/10.1016/j.ccell.2016.09.003

Ewald, J.A., Desotelle, J.A., Wilding, G., Jarrard, D.F., 2010. Therapy-induced senescence in cancer. J. Natl. Cancer Inst. 102, 1536-46. https://doi.org/10.1093/jnci/djq364

Fitzner, B., Müller, S., Walther, M., Fischer, M., Engelmann, R., Müller-Hilke, B., Pützer, B.M., Kreutzer, M., Nizze, H., Jaster, R., 2012. Senescence determines the fate of activated rat pancreatic stellate cells. J. Cell. Mol. Med. 16, 2620-30. https://doi.org/10.1111/j.15824934.2012.01573.x

Flanary, B.E., Streit, W.J., 2004. Progressive telomere shortening occurs in cultured rat microglia, but not astrocytes. Glia 45, 75-88. https://doi.org/10.1002/glia.10301

Frescas, D., Roux, C.M., Aygun-Sunar, S., Gleiberman, A.S., Krasnov, P., Kurnasov, O. V., Strom, E., Virtuoso, L.P., Wrobel, M., Osterman, A.L., Antoch, M.P., Mett, V., Chernova, O.B., Gudkov, A. V., 2017. Senescent cells expose and secrete an oxidized form of membrane-bound vimentin as revealed by a natural polyreactive antibody. Proc. Natl. Acad. Sci. 114, E1668-E1677. https://doi.org/10.1073/pnas.1614661114

Freund, A., Patil, C.K., Campisi, J., 2011. p38MAPK is a novel DNA damage response-independent regulator of the senescence-associated secretory phenotype. EMBO J. 30, 1536-1548. 
https://doi.org/10.1038/emboj.2011.69

Friggeri, A., Banerjee, S., Biswas, S., de Freitas, A., Liu, G., Bierhaus, A., Abraham, E., 2011.

Participation of the receptor for advanced glycation end products in efferocytosis. J. Immunol. 186, 6191-8. https://doi.org/10.4049/jimmunol.1004134

Glück, S., Guey, B., Gulen, M.F., Wolter, K., Kang, T.-W., Schmacke, N.A., Bridgeman, A., Rehwinkel, J., Zender, L., Ablasser, A., 2017. Innate immune sensing of cytosolic chromatin fragments through cGAS promotes senescence. Nat. Cell Biol. 19, 1061-1070. https://doi.org/10.1038/ncb3586

Goel, S., DeCristo, M.J., Watt, A.C., BrinJones, H., Sceneay, J., Li, B.B., Khan, N., Ubellacker, J.M., Xie, S., Metzger-Filho, O., Hoog, J., Ellis, M.J., Ma, C.X., Ramm, S., Krop, I.E., Winer, E.P., Roberts, T.M., Kim, H.-J., McAllister, S.S., Zhao, J.J., 2017. CDK4/6 inhibition triggers anti-tumour immunity. Nature 548, 471-475. https://doi.org/10.1038/nature23465

Hall, B.M., Balan, V., Gleiberman, A.S., Strom, E., Krasnov, P., Virtuoso, L.P., Rydkina, E., Vujcic, S., Balan, K., Gitlin, I., Leonova, K., Polinsky, A., Chernova, O.B., Gudkov, A. V., 2016. Aging of mice is associated with $\mathrm{p} 16$ (Ink4a)- and $\beta$-galactosidase-positive macrophage accumulation that can be induced in young mice by senescent cells. Aging (Albany. NY). 8, 1294-1315. https://doi.org/10.18632/aging.100991

Hazen, S.L., 2008. Oxidized Phospholipids as Endogenous Pattern Recognition Ligands in Innate Immunity. J. Biol. Chem. 283, 15527-15531. https://doi.org/10.1074/jbc.R700054200

Henson, S.M., Lanna, A., Riddell, N.E., Franzese, O., Macaulay, R., Griffiths, S.J., Puleston, D.J., Watson, A.S., Simon, A.K., Tooze, S.A., Akbar, A.N., 2014. p38 signaling inhibits mTORC1independent autophagy in senescent human CD8+ T cells. J. Clin. Invest. 124, 4004-4016. https://doi.org/10.1172/JCI75051

Herranz, N., Gallage, S., Mellone, M., Wuestefeld, T., Klotz, S., Hanley, C.J., Raguz, S., Acosta, J.C., Innes, A.J., Banito, A., Georgilis, A., Montoya, A., Wolter, K., Dharmalingam, G., Faull, P., Carroll, T., Martínez-Barbera, J.P., Cutillas, P., Reisinger, F., Heikenwalder, M., Miller, R.A., Withers, D., Zender, L., Thomas, G.J., Gil, J., 2015. mTOR regulates MAPKAPK2 translation to control the senescence-associated secretory phenotype. Nat. Cell Biol. 17, 1205-1217. https://doi.org/10.1038/ncb3225

Iannello, A., Thompson, T.W., Ardolino, M., Lowe, S.W., Raulet, D.H., 2013. p53-dependent chemokine production by senescent tumor cells supports NKG2D-dependent tumor elimination 
by natural killer cells. J. Exp. Med. 210, 2057-2069. https://doi.org/10.1084/jem.20130783

Itakura, Y., Sasaki, N., Kami, D., Gojo, S., Umezawa, A., Toyoda, M., 2016. N- and O-glycan cell surface protein modifications associated with cellular senescence and human aging. Cell Biosci. 6, 14. https://doi.org/10.1186/s13578-016-0079-5

Joselow, A., Lynn, D., Terzian, T., Box, N.F., 2017. Senescence-Like Phenotypes in Human Nevi. Methods Mol. Biol. 1534, 175-184. https://doi.org/10.1007/978-1-4939-6670-7_17

Jurk, D., Wang, C., Miwa, S., Maddick, M., Korolchuk, V., Tsolou, A., Gonos, E.S., Thrasivoulou, C., Jill Saffrey, M., Cameron, K., von Zglinicki, T., 2012. Postmitotic neurons develop a p21-dependent senescence-like phenotype driven by a DNA damage response. Aging Cell 11, 996-1004. https://doi.org/10.1111/j.1474-9726.2012.00870.x

Kang, C., Xu, Q., Martin, T.D., Li, M.Z., Demaria, M., Aron, L., Lu, T., Yankner, B.A., Campisi, J., Elledge, S.J., 2015. The DNA damage response induces inflammation and senescence by inhibiting autophagy of GATA4. Science (80-. ). 349, aaa5612-aaa5612. https://doi.org/10.1126/science.aaa5612

Kang, T.-W., Yevsa, T., Woller, N., Hoenicke, L., Wuestefeld, T., Dauch, D., Hohmeyer, A., Gereke, M., Rudalska, R., Potapova, A., Iken, M., Vucur, M., Weiss, S., Heikenwalder, M., Khan, S., Gil, J., Bruder, D., Manns, M., Schirmacher, P., Tacke, F., Ott, M., Luedde, T., Longerich, T., Kubicka, S., Zender, L., 2011. Senescence surveillance of pre-malignant hepatocytes limits liver cancer development. Nature 1-5. https://doi.org/10.1038/nature10599

Kim, K.-H., Park, B., Rhee, D.-K., Pyo, S., 2015. Acrylamide Induces Senescence in Macrophages through a Process Involving ATF3, ROS, p38/JNK, and a Telomerase-Independent Pathway. Chem. Res. Toxicol. 28, 71-86. https://doi.org/10.1021/tx500341z

Krizhanovsky, V., Yon, M., Dickins, R.A., Hearn, S., Simon, J., Miething, C., Yee, H., Zender, L., Lowe, S.W., 2008a. Senescence of Activated Stellate Cells Limits Liver Fibrosis. Cell 134, 657-667. https://doi.org/10.1016/j.cell.2008.06.049

Krizhanovsky, V., Yon, M., Dickins, R.A., Hearn, S., Simon, J., Miething, C., Yee, H., Zender, L., Lowe, S.W., 2008b. Senescence of Activated Stellate Cells Limits Liver Fibrosis. Cell 134, 657-667. https://doi.org/10.1016/j.cell.2008.06.049

Kuilman, T., Peeper, D.S., 2009. Senescence-messaging secretome: SMS-ing cellular stress. Nat. Rev. Cancer 9, 81-94. https://doi.org/10.1038/nrc2560

Latorre, E., Birar, V.C., Sheerin, A.N., Jeynes, J.C.C., Hooper, A., Dawe, H.R., Melzer, D., Cox, L.S., 
Faragher, R.G.A., Ostler, E.L., Harries, L.W., 2017. Small molecule modulation of splicing factor expression is associated with rescue from cellular senescence. BMC Cell Biol. 18, 31. https://doi.org/10.1186/s12860-017-0147-7

Li, C., Chai, Y., Wang, L., Gao, B., Chen, H., Gao, P., Zhou, F.-Q., Luo, X., Crane, J.L., Yu, B., Cao, X., Wan, M., 2017. Programmed cell senescence in skeleton during late puberty. Nat. Commun. 8 , 1312. https://doi.org/10.1038/s41467-017-01509-0

Liu, X., Kwon, H., Li, Z., Fu, Y.-X., 2017. Is CD47 an innate immune checkpoint for tumor evasion? J. Hematol. Oncol. 10, 12. https://doi.org/10.1186/s13045-016-0381-z

Lujambio, A., Akkari, L., Simon, J., Grace, D., Tschaharganeh, D.F., Bolden, J.E., Zhao, Z., Thapar, V., Joyce, J.A., Krizhanovsky, V., Lowe, S.W., 2013. Non-cell-autonomous tumor suppression by p53. Cell 153, 449-60. https://doi.org/10.1016/j.cell.2013.03.020

Mackenzie, K.J., Carroll, P., Martin, C.-A., Murina, O., Fluteau, A., Simpson, D.J., Olova, N., Sutcliffe, H., Rainger, J.K., Leitch, A., Osborn, R.T., Wheeler, A.P., Nowotny, M., Gilbert, N., Chandra, T., Reijns, M.A.M., Jackson, A.P., 2017. cGAS surveillance of micronuclei links genome instability to innate immunity. Nature 548, 461-465. https://doi.org/10.1038/nature23449

Malaquin, N., Martinez, A., Rodier, F., 2016. Keeping the senescence secretome under control: Molecular reins on the senescence-associated secretory phenotype. Exp. Gerontol. 82, 39-49. https://doi.org/10.1016/j.exger.2016.05.010

Montes, C.L., Chapoval, A.I., Nelson, J., Orhue, V., Zhang, X., Schulze, D.H., Strome, S.E., Gastman, B.R., 2008. Tumor-Induced Senescent T Cells with Suppressor Function: A Potential Form of Tumor Immune Evasion. Cancer Res. 68, 870-879. https://doi.org/10.1158/0008-5472.CAN-072282

Mosteiro, L., Pantoja, C., Alcazar, N., Marión, R.M., Chondronasiou, D., Rovira, M., FernandezMarcos, P.J., Muñoz-Martin, M., Blanco-Aparicio, C., Pastor, J., Gómez-López, G., De Martino, A., Blasco, M.A., Abad, M., Serrano, M., 2016. Tissue damage and senescence provide critical signals for cellular reprogramming in vivo. Science (80-. ). 354, aaf4445.

https://doi.org/10.1126/science.aaf4445

Muñoz-Espín, D., Cañamero, M., Maraver, A., Gómez-López, G., Contreras, J., Murillo-Cuesta, S., Rodríguez-Baeza, A., Varela-Nieto, I., Ruberte, J., Collado, M., Serrano, M., 2013a. Programmed Cell Senescence during Mammalian Embryonic Development. Cell 155, 1104-1118. https://doi.org/10.1016/j.cell.2013.10.019 
Muñoz-Espín, D., Cañamero, M., Maraver, A., Gómez-López, G., Contreras, J., Murillo-Cuesta, S., Rodríguez-Baeza, A., Varela-Nieto, I., Ruberte, J., Collado, M., Serrano, M., 2013b. Programmed Cell Senescence during Mammalian Embryonic Development. Cell 155, 1104-1118. https://doi.org/10.1016/j.cell.2013.10.019

Muñoz-Espín, D., Serrano, M., 2014. Cellular senescence: from physiology to pathology. Nat. Rev. Mol. Cell Biol. 15, 482-496. https://doi.org/10.1038/nrm3823

Nakad, R., Schumacher, B., 2016. DNA Damage Response and Immune Defense: Links and Mechanisms. Front. Genet. 7, 147. https://doi.org/10.3389/fgene.2016.00147

Ng, K.W., Marshall, E.A., Bell, J.C., Lam, W.L., 2017. cGAS-STING and Cancer: Dichotomous Roles in Tumor Immunity and Development. Trends Immunol. https://doi.org/10.1016/j.it.2017.07.013

Nosbaum, A., Prevel, N., Truong, H.-A., Mehta, P., Ettinger, M., Scharschmidt, T.C., Ali, N.H., Pauli, M.L., Abbas, A.K., Rosenblum, M.D., 2016. Cutting Edge: Regulatory T Cells Facilitate Cutaneous Wound Healing. J. Immunol. 196, 2010-4. https://doi.org/10.4049/jimmunol.1502139

Nyunoya, T., Powers, L.S., Yarovinsky, T.O., Butler, N.S., Monick, M.M., Hunninghake, G.W., 2003. Hyperoxia Induces Macrophage Cell Cycle Arrest by Adhesion-dependent Induction of p21 cip1 and Activation of the Retinoblastoma Protein. J. Biol. Chem. 278, 36099-36106. https://doi.org/10.1074/jbc.M304370200

Oubaha, M., Miloudi, K., Dejda, A., Guber, V., Mawambo, G., Germain, M.-A., Bourdel, G., Popovic, N., Rezende, F.A., Kaufman, R.J., Mallette, F.A., Sapieha, P., 2016. Senescence-associated secretory phenotype contributes to pathological angiogenesis in retinopathy. Sci. Transl. Med. 8, 362ra144. https://doi.org/10.1126/scitranslmed.aaf9440

Qudrat, A., Wong, J., Truong, K., 2017. Engineering mammalian cells to seek senescence-associated secretory phenotypes. J. Cell Sci. 130, 3116-3123. https://doi.org/10.1242/jcs.206979

Rai, P., Onder, T.T., Young, J.J., McFaline, J.L., Pang, B., Dedon, P.C., Weinberg, R.A., 2009. Continuous elimination of oxidized nucleotides is necessary to prevent rapid onset of cellular senescence. Proc. Natl. Acad. Sci. 106, 169-174. https://doi.org/10.1073/pnas.0809834106

Rajagopalan, S., Long, E.O., 2012. Cellular senescence induced by CD158d reprograms natural killer cells to promote vascular remodeling. Proc. Natl. Acad. Sci. 109, 20596-20601. https://doi.org/10.1073/pnas.1208248109

Rakhra, K., Bachireddy, P., Zabuawala, T., Zeiser, R., Xu, L., Kopelman, A., Fan, A.C., Yang, Q., Braunstein, L., Crosby, E., Ryeom, S., Felsher, D.W., 2010. CD4(+) T cells contribute to the 
remodeling of the microenvironment required for sustained tumor regression upon oncogene inactivation. Cancer Cell 18, 485-98. https://doi.org/10.1016/j.ccr.2010.10.002

Ramello, M.C., Tosello Boari, J., Canale, F.P., Mena, H.A., Negrotto, S., Gastman, B., Gruppi, A., Acosta Rodríguez, E. V, Montes, C.L., 2014. Tumor-induced senescent T cells promote the secretion of pro-inflammatory cytokines and angiogenic factors by human monocytes/macrophages through a mechanism that involves Tim-3 and CD40L. Cell Death Dis. 5, e1507. https://doi.org/10.1038/cddis.2014.451

Rebo, J., Causey, K., Zealley, B., Webb, T., Hamalainen, M., Cook, B., Schloendorn, J., 2010. WholeAnimal Senescent Cytotoxic T Cell Removal Using Antibodies Linked to Magnetic Nanoparticles. Rejuvenation Res. 13, 298-300. https://doi.org/10.1089/rej.2009.0964

Reimann, M., Lee, S., Loddenkemper, C., Dörr, J.R., Tabor, V., Aichele, P., Stein, H., Dörken, B., Jenuwein, T., Schmitt, C.A., 2010. Tumor Stroma-Derived TGF- $\beta$ Limits Myc-Driven Lymphomagenesis via Suv39h1-Dependent Senescence. Cancer Cell 17, 262-272. https://doi.org/10.1016/j.ccr.2009.12.043

Ritschka, B., Storer, M., Mas, A., Heinzmann, F., Ortells, M.C., Morton, J.P., Sansom, O.J., Zender, L., Keyes, W.M., 2017. The senescence-associated secretory phenotype induces cellular plasticity and tissue regeneration. Genes Dev. 31, 172-183. https://doi.org/10.1101/gad.290635.116

Ruhland, M.K., Loza, A.J., Capietto, A.-H., Luo, X., Knolhoff, B.L., Flanagan, K.C., Belt, B.A., Alspach, E., Leahy, K., Luo, J., Schaffer, A., Edwards, J.R., Longmore, G., Faccio, R., DeNardo, D.G., Stewart, S.A., 2016. Stromal senescence establishes an immunosuppressive microenvironment that drives tumorigenesis. Nat. Commun. 7, 11762. https://doi.org/10.1038/ncomms11762

Sagiv, A., Biran, A., Yon, M., Simon, J., Lowe, S.W., Krizhanovsky, V., 2013. Granule exocytosis mediates immune surveillance of senescent cells. Oncogene 32, 1971-1977. https://doi.org/10.1038/onc.2012.206

Sagiv, A., Burton, D.G.A., Moshayev, Z., Vadai, E., Wensveen, F., Ben-Dor, S., Golani, O., Polic, B., Krizhanovsky, V., 2016. NKG2D ligands mediate immunosurveillance of senescent cells. Aging (Albany. NY).

Sagiv, A., Krizhanovsky, V., 2013. Immunosurveillance of senescent cells: the bright side of the senescence program. Biogerontology 14, 617-628. https://doi.org/10.1007/s10522-013-9473-0

Schäfer, M., Werner, S., 2008. Cancer as an overhealing wound: an old hypothesis revisited. Nat. Rev. Mol. Cell Biol. 9, 628-38. https://doi.org/10.1038/nrm2455 
Shelton, D.N., Chang, E., Whittier, P.S., Choi, D., Funk, W.D., 1999. Microarray analysis of replicative senescence. Curr. Biol. 9, 939-45.

Spear, P., Wu, M.-R., Sentman, M.-L., Sentman, C.L., 2013. NKG2D ligands as therapeutic targets. Cancer Immun. 13, 8.

Storer, M., Mas, A., Robert-Moreno, A., Pecoraro, M., Ortells, M.C., Di Giacomo, V., Yosef, R., Pilpel, N., Krizhanovsky, V., Sharpe, J., Keyes, W.M., 2013. Senescence Is a Developmental Mechanism that Contributes to Embryonic Growth and Patterning. Cell 155, 1119-1130. https://doi.org/10.1016/j.cell.2013.10.041

Tadokoro, T., Yamamoto, K., Kuwahara, I., Fujisawa, H., Ikekita, M., Taniguchi, A., Sato, T., Furukawa, K., 2006. Preferential reduction of the alpha-2-6-sialylation from cell surface $\mathrm{N}$-glycans of human diploid fibroblastic cells by in vitro aging. Glycoconj. J. 23, 443-52. https://doi.org/10.1007/s10719-006-7152-y

Tasdemir, N., Banito, A., Roe, J.-S., Alonso-Curbelo, D., Camiolo, M., Tschaharganeh, D.F., Huang, C.H., Aksoy, O., Bolden, J.E., Chen, C.-C., Fennell, M., Thapar, V., Chicas, A., Vakoc, C.R., Lowe, S.W., 2016. BRD4 Connects Enhancer Remodeling to Senescence Immune Surveillance. Cancer Discov. 6, 612-29. https://doi.org/10.1158/2159-8290.CD-16-0217

Toso, A., Revandkar, A., Di Mitri, D., Guccini, I., Proietti, M., Sarti, M., Pinton, S., Zhang, J., Kalathur, M., Civenni, G., Jarrossay, D., Montani, E., Marini, C., Garcia-Escudero, R., Scanziani, E., Grassi, F., Pandolfi, P.P., Catapano, C.V., Alimonti, A., 2014. Enhancing Chemotherapy Efficacy in PtenDeficient Prostate Tumors by Activating the Senescence-Associated Antitumor Immunity. Cell Rep. 9, 75-89. https://doi.org/10.1016/j.celrep.2014.08.044

Toussaint, O., Remacle, J., Dierick, J.-F., Pascal, T., Frippiat, C., Royer, V., Magalhacs, J.P., Zdanov, S., Chainiaux, F., 2002. Stress-induced premature senescence: from biomarkers to likeliness of in vivo occurrence. Biogerontology 3, 13-7.

van Deursen, J.M., 2014. The role of senescent cells in ageing. Nature 509, 439-446. https://doi.org/10.1038/nature13193

van Kooyk, Y., Ilarregui, J.M., van Vliet, S.J., 2015. Novel insights into the immunomodulatory role of the dendritic cell and macrophage-expressed C-type lectin MGL. Immunobiology 220, 185-192. https://doi.org/10.1016/j.imbio.2014.10.002

van Tuyn, J., Jaber-Hijazi, F., MacKenzie, D., Cole, J.J., Mann, E., Pawlikowski, J.S., Rai, T.S., Nelson, D.M., McBryan, T., Ivanov, A., Blyth, K., Wu, H., Milling, S., Adams, P.D., 2017. Oncogene- 
Expressing Senescent Melanocytes Up-Regulate MHC Class II, a Candidate Melanoma Suppressor Function. J. Invest. Dermatol. 137, 2197-2207.

https://doi.org/10.1016/j.jid.2017.05.030

Velarde, M.C., Menon, R., 2016. Positive and negative effects of cellular senescence during female reproductive aging and pregnancy. J. Endocrinol. 230, R59-R76. https://doi.org/10.1530/JOE16-0018

Victorelli, S., Passos, J.F., 2017. Telomeres and Cell Senescence - Size Matters Not. EBioMedicine 21, 14-20. https://doi.org/10.1016/j.ebiom.2017.03.027

Walters, H.E., Deneka-Hannemann, S., Cox, L.S., 2016. Reversal of phenotypes of cellular senescence by pan-mTOR inhibition. Aging (Albany. NY). 8, 231-44. https://doi.org/10.18632/aging.100872

Wang, D., DuBois, R.N., 2015. Immunosuppression associated with chronic inflammation in the tumor microenvironment. Carcinogenesis 36, 1085-1093.

https://doi.org/10.1093/carcin/bgv123

Wang, W., Erbe, A.K., Hank, J.A., Morris, Z.S., Sondel, P.M., 2015. NK Cell-Mediated AntibodyDependent Cellular Cytotoxicity in Cancer Immunotherapy. Front. Immunol. 6, 368. https://doi.org/10.3389/fimmu.2015.00368

$\mathrm{Xu}, \mathrm{Y}$, 2006. DNA damage: a trigger of innate immunity but a requirement for adaptive immune homeostasis. Nat. Rev. Immunol. 6, 261-270. https://doi.org/10.1038/nri1804

Xue, W., Zender, L., Miething, C., Dickins, R.A., Hernando, E., Krizhanovsky, V., Cordon-Cardo, C., Lowe, S.W., 2007. Senescence and tumour clearance is triggered by p53 restoration in murine liver carcinomas. Nature 445, 656-660. https://doi.org/10.1038/nature05529

Yang, H., Wang, H., Ren, J., Chen, Q., Chen, Z.J., 2017. cGAS is essential for cellular senescence. Proc. Natl. Acad. Sci. 114, E4612-E4620. https://doi.org/10.1073/pnas.1705499114

Ye, J., Huang, X., Hsueh, E.C., Zhang, Q., Ma, C., Zhang, Y., Varvares, M.A., Hoft, D.F., Peng, G., 2012. Human regulatory T cells induce T-lymphocyte senescence. Blood 120, 2021-2031. https://doi.org/10.1182/blood-2012-03-416040

Yoshida, A., Diehl, J.A., 2015. CDK4/6 inhibitor: from quiescence to senescence. Oncoscience 2, 8967. https://doi.org/10.18632/oncoscience. 256

Yu, S., Li, A., Liu, Q., Li, T., Yuan, X., Han, X., Wu, K., 2017. Chimeric antigen receptor T cells: a novel therapy for solid tumors. J. Hematol. Oncol. 10, 78. https://doi.org/10.1186/s13045-017-0444- 
Yun, M.H., Davaapil, H., Brockes, J.P., 2015. Recurrent turnover of senescent cells during regeneration of a complex structure. Elife 4. https://doi.org/10.7554/eLife.05505

Figure Legends and Tables

Figure 1: "cell ageing" compared with "cell senescence"

Figure 2: Possible mechanisms of senescent cell recognition by macrophages.

Figure 3: Possible immunotherapies targeting senescent cells.

Table 1: Phenotypic changes often associated with cell senescence in vitro. 